PROCEEDINGS OF THE

AMERICAN MATHEMATICAL SOCIETY

Volume 126, Number 11, November 1998, Pages 3337-3346

S 0002-9939(98)04931-4

\title{
MULTIPLIER THEOREMS FOR HERZ TYPE HARDY SPACES
}

\author{
SHANZHEN LU AND DACHUN YANG
}

(Communicated by J. Marshall Ash)

\begin{abstract}
In this paper, the authors establish a multiplier theorem for Herz
\end{abstract} type Hardy spaces.

Let $T_{m}$ be a multiplier operator defined in terms of Fourier transforms by

$$
\widehat{T_{m} f}(\xi)=m(\xi) \widehat{f}(\xi)
$$

for suitable functions $f$. It is well-known that there is a multiplier theorem for $H^{1}\left(\mathbb{R}^{n}\right)$ (see $\left.[\mathrm{FS}]\right)$ : if $\alpha>n / 2$ and

$$
\int_{R<|\xi|<2 R}\left|D^{\beta} m(\xi)\right|^{2} d \xi \leq C R^{n-2|\beta|}, 0<R<\infty,
$$

for all $|\beta| \leq \alpha$, then $T_{m}$ can be extended to be a bounded operator on $H^{1}\left(\mathbb{R}^{n}\right)$. That is, $m$ is a bounded multiplier of $H^{1}\left(\mathbb{R}^{n}\right)$.

Fix a function $\eta \in C_{0}^{\infty}\left(\mathbb{R}^{n}\right)$ with $0 \leq \eta \leq 1, \eta=1$ on $1 / 2 \leq|\xi| \leq 2$ and $\operatorname{supp} \eta \subset\{1 / 4 \leq|\xi| \leq 4\}$. For $\delta>0$, let us denote

$$
m_{\delta}(\xi)=m(\delta \xi) \eta(\xi) .
$$

It is easy to check that (1) is equivalent to

$$
\sup _{\delta}\left\|\widehat{m_{\delta}}\right\|_{K_{2}^{\alpha, 2}\left(\mathbb{R}^{n}\right)}<\infty
$$

where $K_{2}^{\alpha, 2}\left(\mathbb{R}^{n}\right)$ is a non-homogeneous Herz space (see [BS]). By using some embedding relations on Herz spaces, A. Baernstein II and E. T. Sawyer [BS] weakened (2) into

$$
\sup _{\delta}\left\|\widehat{m_{\delta}}\right\|_{K_{1}^{\varepsilon, 1}\left(\mathbb{R}^{n}\right)}<\infty
$$

where $0<\varepsilon<\alpha-\frac{n}{2}$. In fact, this is just a special case of their theorem. In [BS], Baernstein and Sawyer showed that $m$ is a bounded multiplier of $H^{1}\left(\mathbb{R}^{n}\right)$ under an even weaker condition than (3); see Theorem 3b in [BS, page 21].

By using the technique of Herz type Hardy spaces developed by the authors in [LY1]-[LY3] and [Y], in this paper, we shall first establish a multiplier theorem for the homogeneous Herz type Hardy space $H \dot{K}_{q}^{n(1-1 / q), 1}\left(\mathbb{R}^{n}\right)$ which is introduced by the authors of this paper in [LY1]. Then as simple consequences of this theorem, a multiplier theorem for the corresponding non-homogeneous version of the space

Received by the editors April 13, 1995 and, in revised form, April 5, 1997.

1991 Mathematics Subject Classification. Primary 42B15; Secondary 42B30.

Key words and phrases. Herz space, Hardy space, multiplier.

Research was supported by NNSF and SEDF of China. 
$H \dot{K}_{q}^{n(1-1 / q), 1}\left(\mathbb{R}^{n}\right)$ and the special case mentioned above of the multiplier theorem of Baernstein and Sawyer for $H^{1}\left(\mathbb{R}^{n}\right)$ will be deduced.

Now, for the reader's convenience, let us recall the definition of the Herz spaces here. For $k \in \mathbb{Z}$, let $B_{k}=\left\{x \in \mathbb{R}^{n}:|x| \leq 2^{k}\right\}$ and $A_{k}=B_{k} \backslash B_{k-1}$. We also denote by $\chi_{k}$ the characteristic function of the set $A_{k}$.

Definition 1. Let $\alpha \in \mathbb{R}$ and $0<p, q \leq \infty$.

(i) The homogeneous Herz space $\dot{K}_{q}^{\alpha, p}\left(\mathbb{R}^{n}\right)$ is defined in terms of

$$
\|f\|_{\dot{K}_{q}^{\alpha, p}\left(\mathbb{R}^{n}\right)} \equiv\left\{\sum_{k=-\infty}^{\infty} 2^{k \alpha p}\left\|f \chi_{k}\right\|_{L^{q}\left(\mathbb{R}^{n}\right)}^{p}\right\}^{1 / p}
$$

by letting

$$
\dot{K}_{q}^{\alpha, p}\left(\mathbb{R}^{n}\right) \equiv\left\{f \in L_{l o c}^{q}\left(\mathbb{R}^{n} \backslash\{0\}\right):\|f\|_{\dot{K}_{q}^{\alpha, p}\left(\mathbb{R}^{n}\right)}<\infty\right\} .
$$

(ii) The non-homogeneous Herz space $K_{q}^{\alpha, p}\left(\mathbb{R}^{n}\right)$ is defined in terms of

$$
\|f\|_{K_{q}^{\alpha, p}\left(\mathbb{R}^{n}\right)} \equiv\left\{\left\|f \chi_{B_{0}}\right\|_{L^{q}\left(\mathbb{R}^{n}\right)}^{p}+\sum_{k=1}^{\infty} 2^{k \alpha p}\left\|f \chi_{k}\right\|_{L^{q}\left(\mathbb{R}^{n}\right)}^{p}\right\}^{1 / p}
$$

by letting

$$
K_{q}^{\alpha, p}\left(\mathbb{R}^{n}\right) \equiv\left\{f \in L_{l o c}^{q}\left(\mathbb{R}^{n}\right):\|f\|_{K_{q}^{\alpha, p}\left(\mathbb{R}^{n}\right)}<\infty\right\} .
$$

Here the usual modification was made when $p=\infty$.

In what follows, when $p=1,1<q<\infty$ and $\alpha=n(1-1 / q)$, we shall abbreviate $\dot{K}_{q}^{\alpha, p}\left(\mathbb{R}^{n}\right)$ and $K_{q}^{\alpha, p}\left(\mathbb{R}^{n}\right)$, respectively, as $\dot{K}_{q}\left(\mathbb{R}^{n}\right)$ and $A^{q}\left(\mathbb{R}^{n}\right)$. The latter is also said to be the Beurling algebras; see [CL] and [GR].

Definition 2. Let $1<q<\infty$. For $f \in \mathcal{S}^{\prime}\left(\mathbb{R}^{n}\right)$, let $G f$ be the grand maximal function of $f$ (see [FS] for its definition).

(i) The Hardy space $H \dot{K}_{q}\left(\mathbb{R}^{n}\right)$ associated with the Herz space $\dot{K}_{q}\left(\mathbb{R}^{n}\right)$ is defined by

$$
H \dot{K}_{q}\left(\mathbb{R}^{n}\right) \equiv\left\{f \in \mathcal{S}^{\prime}\left(\mathbb{R}^{n}\right): G f \in \dot{K}_{q}\left(\mathbb{R}^{n}\right)\right\} .
$$

In this case, we also define $\|f\|_{H \dot{K}_{q}\left(\mathbb{R}^{n}\right)} \equiv\|G f\|_{\dot{K}_{q}\left(\mathbb{R}^{n}\right)}$.

(ii) The Hardy space $H A^{q}\left(\mathbb{R}^{n}\right)$ associated with the Beurling algebra $A^{q}\left(\mathbb{R}^{n}\right)$ is defined by

$$
H A^{q}\left(\mathbb{R}^{n}\right) \equiv\left\{f \in \mathcal{S}^{\prime}\left(\mathbb{R}^{n}\right): G f \in A^{q}\left(\mathbb{R}^{n}\right)\right\} .
$$

In this case, we also define $\|f\|_{H A^{q}\left(\mathbb{R}^{n}\right)} \equiv\|G f\|_{A^{q}\left(\mathbb{R}^{n}\right)}$.

We remark that $H A^{q}\left(\mathbb{R}^{n}\right)$ was first introduced by Chen and Lau in [CL] for $n=1$, and then by García-Cuerva in [GR] for $n>1$. Obviously, $H \dot{K}_{q}\left(\mathbb{R}^{n}\right)$ is a homogeneous version of $H A^{q}\left(\mathbb{R}^{n}\right)$. Moreover, in [LY1], the authors proved that

$$
H A^{q}\left(\mathbb{R}^{n}\right)=H \dot{K}_{q}\left(\mathbb{R}^{n}\right) \cap L^{q}\left(\mathbb{R}^{n}\right)
$$

and

$$
\|f\|_{H A^{q}\left(\mathbb{R}^{n}\right)} \sim\|f\|_{H \dot{K}_{q}\left(\mathbb{R}^{n}\right)}+\|f\|_{L^{q}\left(\mathbb{R}^{n}\right)} .
$$


It is also well-known that $H A^{q}\left(\mathbb{R}^{n}\right) \varsubsetneqq H \dot{K}_{q}\left(\mathbb{R}^{n}\right) \varsubsetneqq H^{1}\left(\mathbb{R}^{n}\right)$ for any $q \in(1, \infty)$.

Let us now formulate our multiplier theorem for $H \dot{K}_{q}\left(\mathbb{R}^{n}\right)$.

Theorem 1. Let $q \in(1, \infty)$ and $m$ satisfy

$$
M \equiv \sup _{\delta}\left\|\widehat{m_{\delta}}\right\|_{K_{1}^{n(1-1 / q), 1}\left(\mathbb{R}^{n}\right)}<\infty .
$$

Then $m$ is a bounded multiplier of $H \dot{K}_{q}\left(\mathbb{R}^{n}\right)$.

By Corollary 2 in [BS, page 22], we know that if $m$ satisfies the condition of Theorem 1 , then $m$ is a bounded multiplier of $L^{q}\left(\mathbb{R}^{n}\right)$ for $1<q<\infty$. Therefore, from (4), (5) and Theorem 1, we have the following simple corollary.

Corollary 1. Let $q \in(1, \infty)$ and $m$ satisfy (6). Them $m$ is a bounded multiplier of $H A^{q}\left(\mathbb{R}^{n}\right)$.

The proof of Theorem 1 is based on the decomposition characterizations of Herz spaces and Herz type Hardy spaces in terms of central units and central atoms respectively. Let us recall that a function $e(x)$ is said to be a central $(\alpha, q)$ unit of restrict type if $e$ satisfies

i) supp $e \subset B(0, r), r \geq 1$;

ii) $\|e\|_{L^{q}\left(\mathbb{R}^{n}\right)} \leq|B(0, r)|^{-\alpha / n}$.

Lemma 1 ([LY2]). Let $0<\alpha<\infty, 0<p<\infty$ and $1 \leq q<\infty$. Then $f \in$ $K_{q}^{\alpha, p}\left(\mathbb{R}^{n}\right)$ if and only if $f$ can be expressed as

$$
f(x)=\sum_{k=0}^{\infty} \lambda_{k} e_{k}(x)
$$

where each $e_{k}$ is a central $(\alpha, q)$ unit of restrict type supported on $B_{k}$ and $\sum_{k=0}^{\infty}\left|\lambda_{k}\right|^{p}$ $<\infty$. Moreover,

$$
\inf \left\{\left(\sum_{k}\left|\lambda_{k}\right|^{p}\right)^{1 / p}\right\} \sim\|f\|_{K_{q}^{\alpha, p}\left(\mathbb{R}^{n}\right)}
$$

where the infimum is taken over all of the above decompositions of $f$.

Let us now turn to the definition of central atoms. A function $a(x)$ is said to be a central $(1, q)$ atom if $a$ satisfies

(i) $\operatorname{supp} a \subset B(0, r), r>0$;

(ii) $\|a\|_{L^{q}\left(\mathbb{R}^{n}\right)} \leq|B(0, r)|^{1 / q-1}$;

(iii) $\int_{\mathbb{R}^{n}} a(x) d x=0$.

Lemma 2 ([LY3]). Let $1<q<\infty$. Then $f \in H \dot{K}_{q}\left(\mathbb{R}^{n}\right)$ if and only if $f$ can be expressed as

$$
f(x)=\sum_{k=-\infty}^{\infty} \lambda_{k} a_{k}(x)
$$

where each $a_{k}$ is a central $(1, q)$ atom supported on $B_{k}$ and $\sum_{k=-\infty}^{\infty}\left|\lambda_{k}\right|<\infty$. Moreover,

$$
\inf \left\{\sum_{k=-\infty}^{\infty}\left|\lambda_{k}\right|\right\} \sim\|f\|_{H \dot{K}_{q}\left(\mathbb{R}^{n}\right)},
$$

where the infimum is taken over all of the above decompositions of $f$. 
To prove Theorem 1, we still need a lemma. Let $\psi \in \mathcal{S}\left(\mathbb{R}^{n}\right)$, the Schwartz space of functions. In what follows, we let $\widehat{a_{\delta}}(\xi) \equiv \widehat{a}(\delta \xi) \psi(\xi)$.

Lemma 3. Let a be a central $(1, q)$ atom supported on $B(0,1)$ and $b_{j}=\left(\widehat{a_{2^{j}}}\right)^{\vee}$. Then for any given $d>0$, we have the following three facts:

(i) $\left\|b_{j}\right\|_{L^{q}\left(\mathbb{R}^{n}\right)} \leq C 2^{-n j(1-1 / q)}$.

(ii) $\left|b_{j}(x)\right| \leq C_{d} 2^{-n j(1-1 / q)}|x|^{-d}$, for $|x| \geq 2^{j+1}$.

(iii) $\left|b_{j}(x)\right| \leq C_{d} 2^{j}(1+|x|)^{-d}$, for all $x$ and $j \leq 0$.

Proof. Since $1<q<\infty$ and

$$
b_{j}(x)=2^{-n j} \int_{|x-y|<2^{j}} a\left(2^{-j}(x-y)\right) \widehat{\psi}(y) d y,
$$

it follows from the generalized Minkowski inequality that

$$
\begin{aligned}
\left\|b_{j}\right\|_{L^{q}\left(\mathbb{R}^{n}\right)} & \leq 2^{-n j} \int_{\mathbb{R}^{n}}\left\{\int_{\mathbb{R}^{n}}\left|a\left(2^{-j}(x-y)\right)\right|^{q} d x\right\}^{1 / q}|\widehat{\psi}(y)| d y \\
& \leq C 2^{-n j} 2^{n j / q}\|a\|_{L^{q}\left(\mathbb{R}^{n}\right)}\|\widehat{\psi}\|_{L^{1}\left(\mathbb{R}^{n}\right)} \leq C 2^{-n j(1-1 / q)} .
\end{aligned}
$$

Thus, (i) holds. Let us now assume $|x| \geq 2^{j+1}$. Note that $|y| \geq|x| / 2$ and $\widehat{\psi} \in$ $\mathcal{S}\left(\mathbb{R}^{n}\right)$. Then we have

$$
\begin{aligned}
\left|b_{j}(x)\right| & \leq 2^{-n j} \int_{|x-y|<2^{j}}\left|a\left(2^{-j}(x-y)\right)\right| \cdot|\widehat{\psi}(y)| d y \\
& \leq 2^{-n j}\left(\int_{|x-y|<2^{j}}\left|a\left(2^{-j}(x-y)\right)\right|^{q} d y\right)^{1 / q}\left(\int_{|x-y|<2^{j}}|\widehat{\psi}(y)|^{q^{\prime}} d y\right)^{1 / q^{\prime}} \\
& =2^{n j(1 / q-1)}\|a\|_{L^{q}\left(\mathbb{R}^{n}\right)}\left(\int_{|y| \geq|x| / 2}|\widehat{\psi}(y)|^{q^{\prime}} d y\right)^{1 / q^{\prime}} \\
& \leq C_{d} 2^{n j(1 / q-1)}|x|^{-d} .
\end{aligned}
$$

Thus, (ii) also holds. Finally, let us assume $j \leq 0$. Since $\int_{\mathbb{R}^{n}} a(y) d y=0$, we have

$$
b_{j}(x)=\int_{|y|<1} a(y)\left\{\widehat{\psi}\left(x-2^{j} y\right)-\widehat{\psi}(x)\right\} d y .
$$

It follows from the mean value theorem that there exists a $\theta \in(0,1)$ such that

$$
\left|b_{j}(x)\right| \leq \int_{|y|<1}|a(y)| \cdot\left|\left(\nabla_{x} \widehat{\psi}\right)\left(x-\theta 2^{j} y\right)\right| 2^{j}|y| d y,
$$

where $\nabla_{x}=\left(\frac{\partial}{\partial x_{1}}, \cdots, \frac{\partial}{\partial x_{n}}\right)$. Note that $1+\left|x-\theta 2^{j} y\right| \geq(1+|x|) / 2$ and $\widehat{\psi} \in \mathcal{S}\left(\mathbb{R}^{n}\right)$. Then we have

$$
\left|b_{j}(x)\right| \leq C_{d} 2^{j}(1+|x|)^{-d} \int_{|y|<1}|y| \cdot|a(y)| d y \leq C_{d} 2^{j}(1+|x|)^{-d} .
$$

This completes the proof of the lemma.

Proof of Theorem 1. By the decompositions of $H \dot{K}_{q}\left(\mathbb{R}^{n}\right)$ in terms of central atoms, it suffices to prove that the inequality

$$
\left\|T_{m} a\right\|_{H \dot{K}_{q}\left(\mathbb{R}^{n}\right)} \leq C
$$

holds for all central $(1, q)$ atoms $a(x)$. Let $a(x)$ be a central $(1, q)$ atom. Since $M$ is invariant for all dilations of $m$, we may assume supp $a \subset B(0,1)$. In Lemma 3 , 
if we take $\psi \in C_{0}^{\infty}\left(\mathbb{R}^{n}\right)$ such that $\operatorname{supp} \psi \subset\{\xi: 1 / 2 \leq|\xi| \leq 2\}, 0 \leq \psi \leq 1$, and $\sum_{j \in \mathbb{Z}} \psi\left(\xi 2^{-j}\right)=1$, then $\psi \eta=\psi$ and

$$
\begin{aligned}
m(\xi) \widehat{a}(\xi) & =\sum_{j=-\infty}^{\infty} m(\xi) \eta\left(2^{-j} \xi\right) \widehat{a}(\xi) \psi\left(2^{-j} \xi\right) \\
& =\sum_{j=-\infty}^{\infty} m_{2^{j}}\left(2^{-j} \xi\right) \widehat{a_{2^{j}}}\left(2^{-j} \xi\right),
\end{aligned}
$$

where $\widehat{a_{\delta}}(\xi)=\widehat{a}(\delta \xi) \psi(\xi)$. By letting $N_{j} \equiv\left(m_{2^{j}}\right)^{\vee}$, we have

$$
T_{m} a(x)=\sum_{j=-\infty}^{\infty} 2^{n j}\left(N_{j} * b_{j}\right)\left(2^{j} x\right) .
$$

Without loss of generality, we may assume $M=1$. Thus, the inequality $\|{\widehat{m_{\delta}}}_{L_{L^{1}\left(\mathbb{R}^{n}\right)}}$ $\leq 1$ holds for any $\delta>0$. Therefore, it follows from the Hausdorff-Young inequality that $\|m\|_{L^{\infty}\left(\mathbb{R}^{n}\right)} \leq 1$ and

$$
\left\|N_{j}\right\|_{L^{1}\left(\mathbb{R}^{n}\right)} \leq\left\|N_{j}\right\|_{K_{1}^{n(1-1 / q), 1}\left(\mathbb{R}^{n}\right)} \leq 1 .
$$

Let us first prove

$$
\left\|T_{m} a\right\|_{\dot{K}_{q}\left(\mathbb{R}^{n}\right)} \leq C
$$

where $C$ is independent of $a$ and $a$ is a central $(1, q)$ atom with supp $a \subset B(0,1)$. We write

$$
\begin{aligned}
\left\|T_{m} a\right\|_{\dot{K}_{q}\left(\mathbb{R}^{n}\right)} & =\sum_{k=-\infty}^{\infty} 2^{k n(1-1 / q)}\left\|\left(T_{m} a\right) \chi_{k}\right\|_{L^{q}\left(\mathbb{R}^{n}\right)} \\
& =\sum_{k=-\infty}^{2} \cdots+\sum_{k=3}^{\infty} \cdots \equiv I_{1}+I_{2} .
\end{aligned}
$$

Since $m$ is a bounded multiplier of $L^{q}\left(\mathbb{R}^{n}\right)$ by Corollary 2 in [BS, page 22], we have

$$
I_{1} \leq C \sum_{k=-\infty}^{2} 2^{k n(1-1 / q)}\|a\|_{L^{q}\left(\mathbb{R}^{n}\right)} \leq C \sum_{k=-\infty}^{2} 2^{n k(1-1 / q)} \leq C .
$$

On the other hand, by (8), we have

$$
\begin{aligned}
I_{2} & =\sum_{k=3}^{\infty} 2^{k n(1-1 / q)}\left\|\left(T_{m} a\right) \chi_{k}\right\|_{L^{q}\left(\mathbb{R}^{n}\right)} \\
& \leq \sum_{k=3}^{\infty} \sum_{j=-\infty}^{\infty} 2^{n j} 2^{k n(1-1 / q)}\left\|\left(N_{j} * b_{j}\right)\left(2^{j} \cdot\right) \chi_{k}(\cdot)\right\|_{L^{q}\left(\mathbb{R}^{n}\right)} \\
& =\sum_{j=-\infty}^{\infty} \sum_{l=j+3}^{\infty} 2^{n l(1-1 / q)}\left\|\left(N_{j} * b_{j}\right)(\cdot) \chi_{l}(\cdot)\right\|_{L^{q}\left(\mathbb{R}^{n}\right)} \\
& =\sum_{j=-\infty}^{0} \sum_{l=j+3}^{\infty} \cdots+\sum_{j=1}^{\infty} \sum_{l=j+3}^{\infty} \cdots \equiv I_{2,1}+I_{2,2} .
\end{aligned}
$$


Let us first estimate $I_{2,1}$. By Lemma $1, N_{j}$ can be expressed as

$$
N_{j}(x)=\sum_{k=0}^{\infty} \lambda_{k}^{j} e_{k}^{j}(x),
$$

where each $e_{k}^{j}$ is a central $(n(1-1 / q), 1)$ unit of restrict type supported on $B_{k}$ and

$$
\inf \left(\sum_{k=0}^{\infty}\left|\lambda_{k}^{j}\right|\right) \sim\left\|N_{j}\right\|_{K_{1}^{n(1-1 / q), 1}\left(\mathbb{R}^{n}\right)}
$$

Thus,

$$
\begin{aligned}
I_{2,1}= & \sum_{j=-\infty}^{0} \sum_{l=j+3}^{\infty} 2^{n l(1-1 / q)}\left\|\left(N_{j} * b_{j}\right)(\cdot) \chi_{l}(\cdot)\right\|_{L^{q}\left(\mathbb{R}^{n}\right)} \\
\leq & \sum_{j=-\infty}^{0} \sum_{l=j+3}^{\infty} 2^{n l(1-1 / q)} \sum_{k=0}^{\infty}\left|\lambda_{k}^{j}\right| \cdot\left\|\left(e_{k}^{j} * b_{j}\right) \chi_{l}\right\|_{L^{q}\left(\mathbb{R}^{n}\right)} \\
= & \sum_{j=-\infty}^{0} \sum_{l=j+3}^{\infty} 2^{n l(1-1 / q)} \sum_{k=0}^{\max \{l-2,0\}}\left|\lambda_{k}^{j}\right| \cdot\left\|\left(e_{k}^{j} * b_{j}\right) \chi_{l}\right\|_{L^{q}\left(\mathbb{R}^{n}\right)} \\
& +\sum_{j=-\infty}^{0} \sum_{l=j+3}^{\infty} 2^{n l(1-1 / q)} \sum_{k=\max \{l-1,0\}}^{\infty}\left|\lambda_{k}^{j}\right| \cdot\left\|\left(e_{k}^{j} * b_{j}\right) \chi_{l}\right\|_{L^{q}\left(\mathbb{R}^{n}\right)} \\
\equiv & I_{2,1}^{1}+I_{2,1}^{2} .
\end{aligned}
$$

By (iii) in Lemma 3 with $d=n+\varepsilon, 0<\varepsilon<1$, we have

$$
\begin{aligned}
\left\|\left(e_{k}^{j} * b_{j}\right) \chi_{l}\right\|_{L^{q}\left(\mathbb{R}^{n}\right)} & \leq C 2^{j}\left\|e_{k}^{j}\right\|_{L^{1}\left(\mathbb{R}^{n}\right)}\left(\int_{A_{l}}|x|^{-d q} d x\right)^{1 / q} \\
& \leq C 2^{j} 2^{-l(n+\varepsilon)} 2^{l n / q} .
\end{aligned}
$$

Thus, we obtain

$$
\begin{aligned}
I_{2,1}^{1} & \leq C \sum_{j=-\infty}^{0} 2^{j} \sum_{l=j+3}^{\infty} 2^{-l \varepsilon} \sum_{k=0}^{\infty}\left|\lambda_{k}^{j}\right| \\
& \leq C \sum_{j=-\infty}^{0} 2^{j} 2^{-j \varepsilon}\left\|N_{j}\right\|_{K_{1}^{n(1-1 / q), 1}\left(\mathbb{R}^{n}\right)} \\
& \leq C \sum_{j=-\infty}^{0} 2^{j(1-\varepsilon)} \leq C .
\end{aligned}
$$

By (iii) in Lemma 3 again, we have

$$
\begin{aligned}
\left\|\left(e_{k}^{j} * b_{j}\right) \chi_{l}\right\|_{L^{q}\left(\mathbb{R}^{n}\right)} & \leq\left\|e_{k}^{j}\right\|_{L^{1}\left(\mathbb{R}^{n}\right)}\left\|b_{j}\right\|_{L^{q}\left(\mathbb{R}^{n}\right)} \leq C 2^{j}\left|B\left(0,2^{k}\right)\right|^{-(1-1 / q)} \\
& \leq C 2^{j} 2^{-k n(1-1 / q)} .
\end{aligned}
$$


Thus, we obtain

$$
\begin{aligned}
I_{2,1}^{2} & \leq C \sum_{j=-\infty}^{0} 2^{j} \sum_{l=j+3}^{\infty} 2^{n l(1-1 / q)} \sum_{k=\max \{l-1,0\}}^{\infty}\left|\lambda_{k}^{j}\right| 2^{-k n(1-1 / q)} \\
& \leq C \sum_{j=-\infty}^{0} 2^{j} \sum_{k=0}^{\infty}\left|\lambda_{k}^{j}\right| 2^{-k n(1-1 / q)} \sum_{l=-\infty}^{k+1} 2^{n l(1-1 / q)} \\
& \leq C \sum_{j=-\infty}^{0} 2^{j}\left\|N_{j}\right\|_{K_{1}^{n(1-1 / q), 1}\left(\mathbb{R}^{n}\right)} \leq C .
\end{aligned}
$$

Hence, we obtain $I_{2,1} \leq C$.

We now estimate $I_{2,2}$. Let $x \in A_{l}, l \geq j+3$. Then,

$$
\begin{aligned}
\left(N_{j} * b_{j}\right)(x) & =\int_{|y| \leq 2^{l-1}} N_{j}(y) b_{j}(x-y) d y \\
& +\left[\left(N_{j} \chi_{\widetilde{A}_{l}}\right) * b_{j}\right](x)+\int_{|y|>2^{l+2}} N_{j}(y) b_{j}(x-y) d y,
\end{aligned}
$$

where $\widetilde{A}_{l}=A_{l-1} \cup A_{l} \cup A_{l+1}$. Note that if $|y| \leq 2^{l-2}$ and $x \in A_{l}, l \geq j+3$, then $|x-y| \geq 2^{j+1}$ and $|x-y| \geq|x| / 2$. Thus, it follows from (ii) in Lemma 3 that

$$
\left|b_{j}(x-y)\right| \leq C_{d} 2^{-n j(1-1 / q)}|x|^{-d} \leq C_{d} 2^{-n j(1-1 / q)} 2^{-l d} .
$$

Also, note that if $|y|>2^{l+3}$ and $x \in A_{l}, l \geq j+3$, then $|x-y| \geq 2^{j+1}$ and $|x-y| \geq|y| / 2$. Also, it follows from (ii) in Lemma 3 that

$$
\left|b_{j}(x-y)\right| \leq C_{d} 2^{-n j(1-1 / q)}|y|^{-d} \leq C_{d} 2^{-n j(1-1 / q)} 2^{-l d} .
$$

Thus, when $x \in A_{l}, l \geq j+3$, we have

$$
\begin{aligned}
\left|\left(N_{j} * b_{j}\right)(x)\right| & \leq C_{d} 2^{-n j(1-1 / q)} 2^{-l d}\left\|N_{j}\right\|_{L^{1}\left(\mathbb{R}^{n}\right)}+\left|\left(N_{j} \chi_{\widetilde{A}_{l}}\right) * b_{j}(x)\right| \\
& \leq C_{d} 2^{-n j(1-1 / q)-l d}+\left|\left(N_{j} \chi_{\widetilde{A}_{l}}\right) * b_{j}(x)\right| .
\end{aligned}
$$

Applying these estimates and (i) in Lemma 3 with $d=n+\varepsilon$ to $I_{2,2}$, we obtain

$$
\begin{aligned}
I_{2,2}= & \sum_{j=1}^{\infty} \sum_{l=j+3}^{\infty} 2^{n l(1-1 / q)}\left\|\left(N_{j} * b_{j}\right)(\cdot) \chi_{l}(\cdot)\right\|_{L^{q}\left(\mathbb{R}^{n}\right)} \\
\leq & C \sum_{j=1}^{\infty} \sum_{l=j+3}^{\infty} 2^{n l(1-1 / q)} 2^{-n j(1-1 / q)} 2^{-l d} 2^{l n / q} \\
& +\sum_{j=1}^{\infty} \sum_{l=j+3}^{\infty} 2^{l n(1-1 / q)}\left\|\left(N_{j} \chi_{\widetilde{A}_{l}}\right) * b_{j}(\cdot) \chi_{l}(\cdot)\right\|_{L^{q}\left(\mathbb{R}^{n}\right)} \\
\leq & C \sum_{j=1}^{\infty} 2^{-j(d-n / q)}+\sum_{j=1}^{\infty} \sum_{l=j+3}^{\infty} 2^{l n(1-1 / q)}\left\|N_{j} \chi_{\widetilde{A}_{l}}\right\|_{L^{1}\left(\mathbb{R}^{n}\right)}\left\|b_{j}\right\|_{L^{q}\left(\mathbb{R}^{n}\right)} \\
\leq & C+C \sum_{j=1}^{\infty} 2^{-n j(1-1 / q)}\left\|N_{j}\right\|_{K_{1}^{n(1-1 / q), 1}\left(\mathbb{R}^{n}\right)} \leq C+C \sum_{j=1}^{\infty} 2^{-n j(1-1 / q)} \leq C .
\end{aligned}
$$

Now, (9) follows from the above estimates on $I_{1}, I_{2,1}$ and $I_{2,2}$. 
Actually, it is easy to prove that $(9)$ is true for any central $(1, q)$ atom. That is, the inequality

$$
\left\|T_{m} \widetilde{a}\right\|_{\dot{K}_{q}\left(\mathbb{R}^{n}\right)} \leq C
$$

holds for any central $(1, q)$ atom $\widetilde{a}$. In fact, let us assume that $\operatorname{supp} \widetilde{a} \subset B(0, r)$. Obviously, there exists a $k_{0} \in \mathbb{Z}$ such that $2^{k_{0}}<r \leq 2^{k_{0}+1}$. If $I_{1}$ and $I_{2}$ in the proof of (9) are now replaced by

$$
\sum_{k=-\infty}^{k_{0}+2} 2^{k n(1-1 / q)}\left\|\left(T_{m} \widetilde{a}\right) \chi_{k}\right\|_{L^{q}\left(\mathbb{R}^{n}\right)}
$$

and

$$
\sum_{k=k_{0}+3}^{\infty} 2^{k n(1-1 / q)}\left\|\left(T_{m} \widetilde{a}\right) \chi_{k}\right\|_{L^{q}\left(\mathbb{R}^{n}\right)}
$$

respectively, then (10) can be proved by a method similar to that of proving (9).

To prove (7), by the characterization of $H \dot{K}_{q}\left(\mathbb{R}^{n}\right)$ in terms of Riesz transforms (see $[Y]$ ), it suffices to show

$$
\sum_{j=1}^{n}\left\|R_{j}\left(T_{m} a\right)\right\|_{\dot{K}_{q}\left(\mathbb{R}^{n}\right)} \leq C,
$$

where $C$ is independent of $a$ and $R_{j}$ is the $j$-th Riesz transform. Since Riesz transforms are bounded on $H \dot{K}_{q}\left(\mathbb{R}^{n}\right)$ (see $[\mathrm{Y}]$ ), we have

$$
R_{j} a(x)=\sum_{k} \lambda_{k}^{j} a_{k}^{j}(x)
$$

and

$$
\sum_{k}\left|\lambda_{k}^{j}\right| \leq C\left\|R_{j} a\right\|_{H \dot{K}_{q}\left(\mathbb{R}^{n}\right)} \leq C,
$$

where each $a_{k}^{j}$ is a central $(1, q)$ atom and $C$ is independent of $a$. Thus, it follows from (10) that

$$
\begin{aligned}
\sum_{j=1}^{n}\left\|R_{j}\left(T_{m} a\right)\right\|_{\dot{K}_{q}\left(\mathbb{R}^{n}\right)} & =\sum_{j=1}^{n}\left\|\sum_{k} \lambda_{k}^{j}\left(T_{m} a_{k}^{j}\right)\right\|_{\dot{K}_{q}\left(\mathbb{R}^{n}\right)} \\
& \leq \sum_{j=1}^{n} \sum_{k}\left|\lambda_{k}^{j}\right| \cdot\left\|T_{m} a_{k}^{j}\right\|_{\dot{K}_{q}\left(\mathbb{R}^{n}\right)} \leq C \sum_{j=1}^{n} \sum_{k}\left|\lambda_{k}^{j}\right| \leq C .
\end{aligned}
$$

Thus, (11) holds. This completes the proof of Theorem 1.

Let us now point out that if a linear operator $T$ commutes with translations, then the boundedness of $T$ on $H \dot{K}_{q}\left(\mathbb{R}^{n}\right)$ implies its boundedness on $H^{1}\left(\mathbb{R}^{n}\right)$. Precisely, we have

Theorem 2. Let $T$ be a linear operator that commutes with translations. If $T$ is bounded on $H \dot{K}_{q}\left(\mathbb{R}^{n}\right), 1<q<\infty$, then $T$ is also bounded on $H^{1}\left(\mathbb{R}^{n}\right)$. 
Proof. By the atomic decomposition of $H^{1}\left(\mathbb{R}^{n}\right)$ (see $[\mathrm{CW}]$ ), it suffices to prove that the inequality

$$
\|T a\|_{H^{1}\left(\mathbb{R}^{n}\right)} \leq C
$$

holds for any $(1, q)$ atom $a$. Let $a(x)$ be a $(1, q)$ atom supported on $B\left(x_{0}, r\right)$. That is, $a(x)$ satisfies the following conditions: $\operatorname{supp} a \subset B\left(x_{0}, r\right),\|a\|_{L^{q}\left(\mathbb{R}^{n}\right)} \leq$ $\left|B\left(x_{0}, r\right)\right|^{1 / q-1}$, and $\int_{\mathbb{R}^{n}} a(x) d x=0$. Let $\bar{a}(x) \equiv \tau_{-x_{0}} a(x)=a\left(x+x_{0}\right)$. It is easy to see that $\bar{a}$ is a central $(1, q)$ atom supported on $B(0, r)$. Thus, from the conditions of the theorem, it follows that

$$
\|T \bar{a}\|_{H \dot{K}_{q}\left(\mathbb{R}^{n}\right)} \leq C
$$

where $C$ is independent of $\bar{a}$. Since $T$ commutes with translations, we have

$$
\begin{aligned}
\left\|\tau_{-x_{0}} T a\right\|_{H^{1}\left(\mathbb{R}^{n}\right)} & \leq\left\|\tau_{-x_{0}} T a\right\|_{H \dot{K}_{q}\left(\mathbb{R}^{n}\right)}=\left\|T \tau_{-x_{0}} a\right\|_{H \dot{K}_{q}\left(\mathbb{R}^{n}\right)} \\
& =\|T \bar{a}\|_{H \dot{K}_{q}\left(\mathbb{R}^{n}\right)} \leq C .
\end{aligned}
$$

Thus, $\tau_{-x_{0}} T a \in H^{1}\left(\mathbb{R}^{n}\right)$ and

$$
\tau_{-x_{0}} T a(x)=\sum_{j} \lambda_{j} a_{j}(x)
$$

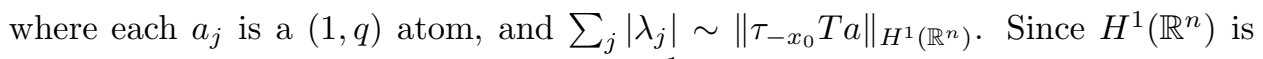
translation invariant, we then have $T a \in H^{1}\left(\mathbb{R}^{n}\right)$ and

$$
\|T a\|_{H^{1}\left(\mathbb{R}^{n}\right)} \leq \sum_{j}\left|\lambda_{j}\right| \leq C
$$

This finishes the proof of Theorem 2 .

Note that if $q \in(1, \infty)$, then $\alpha=n(1-1 / q) \in(0, n)$. As a simple corollary of Theorem 1 and Theorem 2, we have

Corollary 2. Let $0<\varepsilon<n$. If $m$ satisfies

$$
\sup _{\delta}\left\|\widehat{m_{\delta}}\right\|_{K_{1}^{\varepsilon, 1}\left(\mathbb{R}^{n}\right)}<\infty
$$

then $m$ is a bounded multiplier of $H^{1}\left(\mathbb{R}^{n}\right)$.

Finally, we point out that it is still an open problem whether (6) is a necessary condition for an $L^{\infty}\left(\mathbb{R}^{n}\right)$ function $m$ to be a bounded multiplier of $H \dot{K}_{q}\left(\mathbb{R}^{n}\right)$ in any sense (see $[\mathrm{BS}])$. And we will discuss the similar problems of multipliers on general Herz type Hardy spaces $H \dot{K}_{q}^{\alpha, p}\left(\mathbb{R}^{n}\right)$ in a future paper.

\section{ACKNOWLEDGMent}

We thank the referee very much for his many helpful comments. We also thank Professor J. Marshall Ash very much for his kindness and enthusiasm. 


\section{REFERENCES}

[BS] A. Baernstein II and E. T. Sawyer, Embedding and Multiplier Theorem for $H^{p}\left(\mathbb{R}^{n}\right)$, Memoirs of Amer. Math. Soc., vol. 53, no. 318, Amer. Math. Soc., Providence R. I., 1985. MR 86g: 42036

[CL] Y. Z. Chen and K. S. Lau, On some new classes of Hardy spaces, J. Funct. Anal. 84 (1989), 255-278. MR 90f:46059

[CW] R. R. Coifman and G. Weiss, Extensions of Hardy spaces and their use in analysis, Bull. Amer. Math. Soc. 83 (1977), 569-645. MR 56:6264

[FS] C. Fefferman and E. M. Stein, $H^{p}$ spaces of several variables, Acta Math. 129 (1972), 137-193. MR 56:6263

[GR] J. García-Cuerva, Hardy spaces and Beurling algebras, J. London Math. Soc. (2) 39 (1989), 499-513. MR 90i:42032

[LY1] S. Z. Lu and D. C. Yang, The Littlewood-Paley function and $\varphi$-transform characterizations of a new Hardy space $\mathrm{HK}_{2}$ associated with the Herz space, Studia Math. 101 (1992), 285-298. MR 93b:42031

[LY2] S. Z. Lu and D. C. Yang, The decomposition of the weighted Herz spaces on $\mathbb{R}^{n}$ and its applications, Sci. in China (Ser. A) 38 (1995), 147-158. MR 96c:46026

[LY3] S. Z. Lu and D. C. Yang, The weighted Herz-type Hardy space and its applications, Sci. in China (Ser. A) 38 (1995), 662-673. MR 96i:42018

[Y] D. C. Yang, Some Hardy Spaces Associated with the Herz Spaces, Doctoral Dissertation, Beijing Normal University, Beijing, 1992.

Department of Mathematics, Beijing Normal University, Beijing 100875, People's Republic of China

E-mail address: lusz@bnu.edu.cn

E-mail address: dcyang@bnu.edu.cn 